\title{
OFFICERS PAST AND PRESENT
}

\section{PRESIDENT}

\begin{tabular}{|c|c|c|c|}
\hline $\begin{array}{l}\text { H. VAN AMRINGE, } \\
\text { MORY MCCLINTOCK, } \\
\text { W. HILL, } \\
\text { IMON NEWCOMB, } \\
\text { S. WoODWARD, }\end{array}$ & $\begin{array}{l}1888-1890, \\
1890-1894, \\
1894-1896, \\
1896-1898, \\
1898-1900,\end{array}$ & $\begin{array}{l}\text { Eliakim H. MoORE, } \\
\text { THomas S. Fiske, } \\
\text { W. F. OsGood, } \\
\text { H. S. White, } \\
\text { Maxime Bôcher, }\end{array}$ & $\begin{array}{l}1900-1902, \\
1902-1904, \\
1904-1906, \\
1906-1908, \\
1908-\end{array}$ \\
\hline \multicolumn{4}{|c|}{ VICE-PRESIDENTS } \\
\hline $\begin{array}{l}\text { MORY MOCLINTOCK, } \\
\text { K. REES, } \\
\text { ENRY B. FINE, } \\
\text { W. HILL, } \\
\text { A. NEWTON, } \\
\text { S. WOODWARD, } \\
\text { LIAKIM H. MOORE, } \\
\text { HOMAS S. FISKE, } \\
\text { ENRY S. WHITE, } \\
\text { LAXIME BÔCHER, } \\
\text { RANK MORLEY, } \\
\text { T. F. OSGOOD, } \\
\text { LEXANDER ZIWET, }\end{array}$ & $\begin{array}{l}1889-1890, \\
1890-1891, \\
1891-1893, \\
1893-1894, \\
1894-1896, \\
1896-1898, \\
1897-1900, \\
1898-1901, \\
1900-1901, \\
1901-1902, \\
1901-1902, \\
1902-1903, \\
1902-1903,\end{array}$ & $\begin{array}{l}\text { OSKAR BoLZA, } \\
\text { J. M. VAN VLECK, } \\
\text { E. W. BROWN, } \\
\text { JAMES PIERPONT, } \\
\text { CHARLOTTE A. SCOTT, } \\
\text { IRVING STRINGHAM, } \\
\text { HEINRICH MASCHKE, } \\
\text { P. F. SMITH, } \\
\text { JAMES HARKNESS, } \\
\text { G. A. MILLER, } \\
\text { EDWARD KASNER, } \\
\text { E. B. VAN VLECK, } \\
\text { L. E. DICKSON, }\end{array}$ & $\begin{array}{l}1903-1904, \\
1903-1904, \\
1904-1905, \\
1904-1905, \\
1905-1906, \\
1905-1906, \\
1906-1907, \\
1906-1907, \\
1907-1908, \\
1907-1908, \\
1908-1909, \\
1908-1909, \\
1909-\quad,\end{array}$ \\
\hline
\end{tabular}

J. I. Hutchinson, 1909- .

\section{SECRETARY}

Thomas S. Fiske, 1888-1895, F. N. Cole, 1895-

TREASURER

Thomas S. Fiske, 1888-1891, Harold Jacoby, 1896-1899, HAROLD JACOBY, 1891-1894, W. S. DeNNETT, 1899-1907, R. S. WOODWARD, 1894-1896, J. H. TANNER, 1907- .

\section{LIBRARIAN}

\begin{tabular}{|c|c|c|c|}
\hline $\begin{array}{l}\text { D. A. Murray, } \\
\text { Gustave Legras, }\end{array}$ & $\begin{array}{l}\text { 1892-1893, } \\
\text { 1893-1894, } \\
\text { D. E. SMITH, }\end{array}$ & $\begin{array}{l}\text { EDWARD L. StableR, } \\
\text { POMEROY LAdUE, } \\
\text { 1901- } \quad .\end{array}$ & $\begin{array}{l}\text { 1894-1895, } \\
1895-1901,\end{array}$ \\
\hline \multicolumn{4}{|c|}{ COMMITTEE OF PUBLICATION } \\
\hline $\begin{array}{l}\text { THOMAS S. FISKE, } \\
\text { HAROID JACOBY, } \\
\text { ALEXANDER ZIWET, } \\
\text { FRANK MORLEY, }\end{array}$ & $\begin{array}{l}1891-1899, \\
1891-1893, \\
1893-1907, \\
1894-1898,\end{array}$ & $\begin{array}{l}\text { F. N. ColE, } \\
\text { FrANK MorLeY, } \\
\text { D. E. SMITH, } \\
\text { VIRGIL SNYDER, }\end{array}$ & $\begin{array}{l}1898- \\
1899-1902, \\
1902-1909, \\
1907-,\end{array}$ \\
\hline
\end{tabular}

